\title{
Field and anisotropy driven transformations of spin spirals in cubic skyrmion hosts
}

\author{
A. O. Leonov, ${ }^{1,2, *}$ C. Pappas ${ }^{3, \dagger}$ and I. Kézsmárki ${ }^{4, *}$ \\ ${ }^{1}$ Department of Chemistry, Faculty of Science, Hiroshima University Kagamiyama, Higashi Hiroshima, Hiroshima 739-8526, Japan \\ ${ }^{2}$ IFW Dresden, Postfach 270016, D-01171 Dresden, Germany \\ ${ }^{3}$ Faculty of Applied Sciences, Delft University of Technology, Mekelweg 15, 2629JB Delft, Netherlands \\ ${ }^{4}$ Experimental Physics V, Center for Electronic Correlations and Magnetism, University of Augsburg, 86135 Augsburg, Germany
}

(Received 7 September 2020; revised 16 November 2020; accepted 18 November 2020; published 17 December 2020)

\begin{abstract}
We discuss distinctive features of spiral states in bulk chiral magnets such as $\mathrm{MnSi}$ and $\mathrm{Cu}_{2} \mathrm{OSeO}_{3}$ that stem from the effect of the cubic magnetocrystalline anisotropy. First of all, at both the helical-to-conical and the conical-to-ferromagnetic transitions, taking place at $H_{c 1}$ and $H_{c 2}$, respectively, the cubic anisotropy leads to reversible or irreversible jumplike reorientations of the spiral wave vectors. The subtle interplay between the easy and hard anisotropy axes gives rise to a phase transition between elliptically distorted conical states almost without any detectable change in the period. We show that the competition between on-site cubic and exchange anisotropy terms can also lead to oblique spiral states. Our work gives clear directions for further experimental studies to reveal theoretically predicted spiral states in cubic helimagnets beyond the aforementioned wellestablished states and thus it can help to understand the magnetic phase diagram of these archetypal skyrmion hosts. In addition, we show that properties of isolated skyrmions such as interskyrmion attraction, orientation, and/or nucleation are also rooted in the properties of host spiral states, in which skyrmions are stabilized.
\end{abstract}

DOI: 10.1103/PhysRevResearch.2.043386

\section{INTRODUCTION}

Following the pioneering theoretical works of Dzyaloshinskii [1,2] and the experimental findings in the 1980s, where the magnetic phase diagram of $\mathrm{MnSi}$ was studied by different techniques and the results were summarized by Kadowaki [3], chiral helimagnetism [4] has experienced an increasing interest over the last years. This is mainly due to the the discovery of skyrmions [5-7] - two-dimensional localized whirls of the magnetization, which may be used in future information storage and data processing devices. Presently the field of skyrmionics encompasses activities to create real prototypes of skyrmion-based devices in nanosystems such as nanolayers $[6,7]$, nanowires [8], or nanodots $[9,10]$, exploiting the small size, topological protection, and ease with which skyrmions can be manipulated by electric currents. This swerve from bulk cubic helimagnets to nanosystems, witnessed over the last years, was stimulated by the reduced skyrmion stability in the former case, where skyrmions appear forming hexagonal lattices in a small pocket of the temperature-magnetic field phase diagram just below the transition temperature $T_{C}$, the so-called $A$ phase.

\footnotetext{
*leonov@ @iroshima-u.ac.jp

$\dagger$ c.pappas@tudelft.nl

*istvan.kezsmarki@physik.uni-augsburg.de
}

Published by the American Physical Society under the terms of the Creative Commons Attribution 4.0 International license. Further distribution of this work must maintain attribution to the author(s) and the published article's title, journal citation, and DOI.
Recent experimental findings, however, revealed a vast area of low-temperature skyrmion stability in the bulk insulating cubic helimagnet $\mathrm{Cu}_{2} \mathrm{OSeO}_{3}$, when the magnetic field is applied along the easy $\langle 001\rangle$ crystallographic axis $[11,12]$. Remarkably, the theoretical explanation of this phenomenon is mainly based on the effect of single-ion cubic anisotropy on one-dimensional spiral states [13,14]. In fact, the ideal magnetization rotation in the conical phase, the main competitor of skyrmions, can be impaired by the easy and hard anisotropy axes for specific directions of the magnetic field. Through this mechanism, skyrmions, which are more resilient to anisotropy-induced deformations, due to their twodimensional nature, gain stability over extended regions of the phase diagram. In general, it was shown that the cubic anisotropy has a direct impact on the spiral states and thus indirectly is at the origin of many remarkable phenomena for skyrmions.

The experimental findings provide hints that the cubic anisotropy in $\mathrm{Cu}_{2} \mathrm{OSeO}_{3}$ initiates the nucleation of thermodynamically stable skyrmions via two different mechanisms [13]: either via rupture formation of metastable spiral states with wave vectors perpendicular to the field, or via torons [15]. The latter are localized particles consisting of two Bloch points at finite distance and a convex-shaped skyrmion stretching between them. In $\mathrm{Cu}_{2} \mathrm{OSeO}_{3}$ they can be generated by the inhomogeneous magnetic environment provided by the smooth transition between tilted spiral domains, which appear when the magnetic field is applied along the easy $\langle 001\rangle$ crystallographic axis [11]. This tilted spiral phase constitutes a major deviation from the generic phase diagram of cubic chiral magnets $[16,17]$ and is stabilized by a competition between on-site cubic and exchange anisotropies. This new 
phase may also give rise to new topological magnetic defects, such as isolated skyrmions, with interesting static and dynamic properties. Moreover, versatile skyrmion phases have been recently identified both at and above room temperature in a family of cubic chiral magnets: $\beta$-Mn-type Co-Zn-Mn alloys with a different chiral space group from that of B20 compounds [18].

Here we undertake a systematic study of the effects imposed by cubic anisotropy on one-dimensional spiral states. First, we show that cubic anisotropy accounts for the reorientation processes of spiral states that occur not only at the low critical field $H_{c 1}$, but also near the transition into the saturated state $H_{c 2}$, an effect that might at a first sight seem counterintuitive. Furthermore, we show that the existence of easy and hard anisotropy axes distorts elliptically the magnetization rotation and this effect may stabilize new spiral phases with no changes of the spiral orientation or period, but with detectable transitions between them. Our theoretical approach explains the behavior of the bulk helimagnet $\mathrm{Cu}_{2} \mathrm{OSeO}_{3}$, or $\mathrm{MnSi}$, and provides a mechanism through which it is possible to generate thermodynamically stable skyrmionic spin textures over large fractions of their phase diagrams.

\section{PHENOMENOLOGICAL THEORY OF SPIRAL STATES IN CUBIC HELIMAGNETS}

\section{A. The general micromagnetic energy functional}

Within the phenomenological theory introduced by Dzyaloshinskii [1] the magnetic energy density of a noncentrosymmetric ferromagnet with spatially dependent magnetization $\mathbf{M}$ can be written as

$$
W(\mathbf{M})=\underbrace{A \sum_{i, j}\left(\frac{\partial m_{j}}{\partial x_{i}}\right)^{2}+D w_{D}(\mathbf{M})-\mathbf{M} \cdot \mathbf{H}}_{W_{0}(\mathbf{M})}+W_{a}(\mathbf{m}),
$$

where $A$ and $D$ are coefficients of exchange and the Dzyaloshinskii-Moriya interaction (DMI), $\mathbf{H}$ is an applied magnetic field, and $x_{i}$ are the Cartesian components of the spatial variable. $w_{D}$ is composed of Lifshitz invariants $\mathcal{L}_{i, j}^{(k)}=$ $M_{i} \partial M_{j} / \partial x_{k}-M_{j} \partial M_{i} / \partial x_{k}$ that are energy terms involving first derivatives of the magnetization with respect to the spatial coordinates. In the following, all calculations will be done for cubic helimagnets with $w_{D}=\mathbf{m} \cdot \nabla \times \mathbf{m}$ although the results may be applied for magnets with other symmetry classes [19] including different combinations of Lifshitz invariants. $W_{0}(\mathbf{M})$ includes only basic interactions essential to stabilize skyrmion and helical states and specifies their most general features attributed to all chiral ferromagnets. Next we discuss the physics governed by these dominant interactions. The effects of anisotropy $W_{a}(\mathbf{m})$, usually representing a smaller energy scale, will be discussed later.

\section{B. One-dimensional spiral modulations within the isotropic theory}

In chiral magnets the Dzyaloshinskii-Moriya interactions $w_{D}$ play a crucial role in destabilizing the homogeneous ferromagnetic (FM) arrangement and twisting it into a helix. At zero magnetic field the helices are single-harmonic modes forming the global minimum of the functional $W_{0}(\mathbf{M})$ [1], $\mathbf{M}=M_{s}\left[\mathbf{n}_{1} \cos (\mathbf{q} \cdot \mathbf{r})+\mathbf{n}_{2} \sin (\mathbf{q} \cdot \mathbf{r})\right] . \mathbf{n}_{1}, \mathbf{n}_{2}$ are the unit vectors in the plane of the magnetization rotation orthogonal to the wave vector $\mathbf{q}=\mathbf{n}_{3} / L_{D}$ with $\mathbf{n}_{3} \perp \mathbf{n}_{2} \perp \mathbf{n}_{1}$ (i.e., $\mathbf{n}_{1}, \mathbf{n}_{2}$, and $\mathbf{n}_{3}$ are three mutually orthogonal unit vectors). $L_{D}$ is proportional to the ratio of the counteracting exchange and Dzyaloshinskii constants and introduces a fundamental length characterizing the periodicity of chiral modulations in chiral magnets, $L_{D}=A / D$.

The helical modulations have a fixed rotation sense determined by the sign of Dzyaloshinskii-Moriya constant $D$ and are continuously degenerate with respect to propagation directions of the helical modulations in space. An applied magnetic field lifts this degeneracy and stabilizes two types of one-dimensional modulations: cones and helicoids with propagation directions parallel and perpendicular to the applied magnetic field, respectively.

For helicoids, analytical solutions for the polar angle $\theta(x)$ of the magnetization written in spherical coordinates, $\mathbf{M}=M_{s}(\sin \theta(x) \cos \psi, \sin \theta(x) \sin \psi, \cos \theta(x))$, are derived by solving a pendulum equation $A d^{2} \theta / d x^{2}-H \cos \theta=0$. Such solutions are expressed as a set of elliptical functions [1] and describe a gradual expansion of the helicoid period with increased magnetic field (see the set of angular profiles $\theta(x)$, e.g., in Fig. 4.1 of Ref. [20]). In a sufficiently high magnetic field $H_{H}$ the helicoid infinitely expands and transforms into a system of isolated noninteracting $2 \pi$-domain walls (kinks) separating domains with the magnetization along the applied field [1,21]. The dimensionless value of this critical field is $h_{H}=H_{H} / H_{D}=\pi^{2} / 8=0.30843$ with $H_{D}=D^{2} / A M$. The azimuthal angle $\psi$, on the contrary, is fixed by the different forms of the Lifshitz invariants. In particular $\psi=\pi / 2$ for Bloch helicoids in cubic helimagnets and $\psi=0$ for Néel helicoids or cycloids in polar skyrmion hosts with $C_{n v}$ symmetry such as the lacunar spinels $\mathrm{GaV}_{4} \mathrm{~S}_{8}$ [22,23], $\mathrm{GaV}_{4} \mathrm{Se}_{8}$ [24], $\mathrm{GaMo}_{4} \mathrm{~S}_{8}$ [25], or $\mathrm{VOSe}_{2} \mathrm{O}_{5}$ [26]. In this latter class of materials, the polar axial symmetry gives rise to Lifshitz invariants different from that of the cubic helimagnets [Eq. (1)] and the modulation vectors are restricted to the plane perpendicular to the high-symmetry axis.

The conical state combines the properties of the ferromagnetic and the helical states as a compromise between the Zeeman and DM energies. The conical spirals retain their single-harmonic character with $\psi=z / 2 L_{D}$ and $\cos \theta=$ $|\mathbf{H}| / 2 H_{D}$. The magnetization component along the applied field has a fixed value $M_{\perp}=M \cos \theta=M H / 2 H_{D}$ and the magnetization vector $\mathbf{M}$ rotates within the surface of a cone. The critical value $h_{d}=2 H_{D}$ marks the saturation field of the conical state. For the functional $W_{0}(\mathbf{M})$ in Eq. (1) the conical phase is the global minimum over the whole range of applied fields where modulated states exist $\left(0<h<h_{d}\right)$, whereas helicoids and skyrmion lattices can exist only as metastable states.

\section{SPIRAL STATES IN HELIMAGNETS WITH CUBIC ANISOTROPY}

In the following we discuss the influence of the cubic anisotropy on the one-dimensional modulated states. $W_{a}(\mathbf{m})$ 

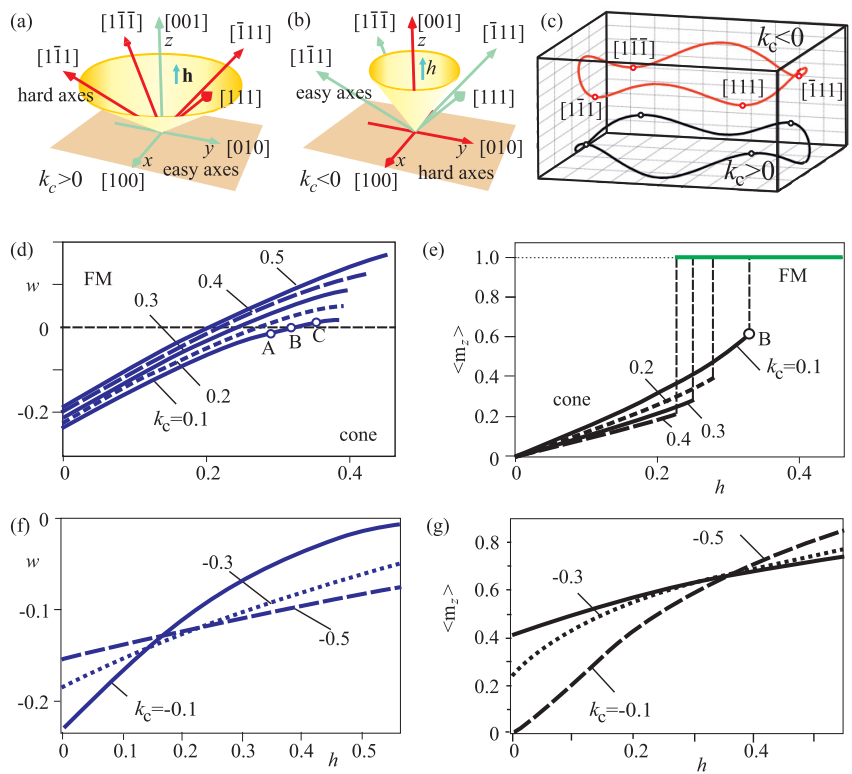

FIG. 1. Schematic representations of the magnetization rotation in the conical phase in the presence of cubic anisotropy with $k_{c}>0$ (a) and $k_{c}<0$ (b) shown together with the magnetization traces in a space (c). Depending on the orientation of the cone propagation direction $(z)$ with respect to the easy (green arrows) and hard (red arrows) anisotropy axes and the applied magnetic field ( $h||[001])$ the energy density of the conical phase can be increased or reduced. This phase undergoes a first-order phase transition into the ferromagnetic state at the critical field $h_{c 2}$ for $k_{c}>0$ and $\mathbf{h}||[001]$. This is seen at the energy density difference between the conical and ferromagnetic phases, depicted in (d), and the magnetization curves shown in (e). The jumps in (e) occur at the field value where the energy density difference in (d) becomes zero, e.g., in the point $B$ for $k_{c}=0.1$. For $k_{c}<0$ and $\mathbf{h} \|[001]$ (f), (g), the mutual arrangement of easy and hard anisotropy axes, shown in (b), underlies a second-order phase transition.

in Eq. (1) can be written as

$$
W_{a}(\mathbf{m})=K_{c}\left(m_{x}^{2} m_{y}^{2}+m_{x}^{2} m_{z}^{2}+m_{y}^{2} m_{z}^{2}\right),
$$

where $K_{c}$ is the coefficient of the cubic magnetic anisotropy and the vector field of the magnetization is given by $\mathbf{m}(\mathbf{r})=$ $\mathbf{M}(\mathbf{r}) / M_{s}$ under the constraint $|\mathbf{m}|=1$.

Generically, there are only small energy differences between various modulated states including skyrmions. On the other hand, weaker energy contributions, such as $W_{a}(\mathbf{m})$, impose distortions on the solutions of the model (1) which reflect the crystallographic symmetry and the values of magnetic interactions of the different chiral magnets. Thus, it is essential to recognize that these weaker interactions play a crucial role in determining the stability limits of the different modulated states on the corresponding phase diagrams.

In the case of single-ion cubic anisotropy, the energy of the conical phase can be either increased or decreased depending on the mutual arrangement of the easy anisotropy axes and the propagation direction of the cones. A good starting point is a simple analysis of the homogeneous state in a system with the easy cubic anisotropy axis parallel to the applied magnetic field as described in Ref. [27] and shown in Fig. 2. In this case, the manifold of energy extrema provides a hint on the distortions of the conical state. The rotation of the magnetization becomes in tune with a complex landscape of the energy term associated with the cubic anisotropy (Fig. 1). The direction of the wave vector $\mathbf{q}$ itself is also specified by the competing Zeeman and cubic anisotropy energies. These basic concepts are clearly manifested, in particular, in the critical fields at the standard phase diagrams of bulk cubic helimagnets such as $\mathrm{MnSi}$ and $\mathrm{Cu}_{2} \mathrm{OSeO}_{3}$ : the critical fields $H_{c 1}$, where oblique spirals align along the field, and $H_{c 2}$, where the conical phase undergoes a phase transition to the ferromagnetic state.

\section{A. The conical-to-ferromagnetic phase transition at the critical field $\boldsymbol{H}_{\boldsymbol{c} 2}$}

In this section we present solutions for spiral states, which we obtained by exact numerical minimization of the energy functional, given by Eq. (1), including the cubic anisotropy $K_{c}$ (2). For this purpose, we consider the dimensionless anisotropy constant, $k_{c}=K_{c} A / D^{2}$. The magnetic field $\mathbf{H}$ is applied along the high-symmetry directions [110], [111], and [001]. The energy density is measured in nondimensional units, $w(\mathbf{m})=W(\mathbf{M}) / H_{D} M$.

\section{First- or second-order phase transitions between the conical and the ferromagnetic state}

In the present section, we consider the solutions for the conical state in the field applied along [001] or [111] for both signs of $k_{c}$. As will become evident later, first-order phase transitions occur when the magnetic field is applied along the easy anisotropy axes. In this case, on its way to saturation, the magnetization is forced to embrace hard anisotropy axes, as shown in Figs. 1(a) and 1(c). This is an energetically unfavorable configuration that forces a discontinuous transition of the magnetization toward the magnetic field. On the contrary, if the magnetic field points toward a hard anisotropy axis, the minima of the cubic anisotropy gradually align along the field and underlie the continuous second-order phase transition between the conical and the ferromagnetic state, as illustrated by Figs. 1(b) and 1(c).

In the following, we carry out a comprehensive analysis of the magnetization rotation in the conical phase by considering the surface energy plots shown in the first row of Fig. 2, and the three-dimensional polar plots of the cubic anisotropy energy depicted in the second row of Fig. 2. The different topologies of these surface plots underly the particular types of the phase transitions.

For $k_{c}>0$ and $h=0$ [Fig. 2(a)] the equilibrium states of the homogeneous state correspond to the easy axes of cubic anisotropy oriented along the $\langle 001\rangle$ crystallographic directions. Maxima of the cubic anisotropy energy correspond to the hard $\langle 111\rangle$ directions. Let us consider the helical monodomain state where the magnetization for $h=0$ rotates in the plane (001). While rotating, the magnetization leaves one energy minimum corresponding to $\langle 001\rangle$ directions and, rotating through the saddle point between hard axes $\langle 111\rangle$, gets into another energy minimum along a $\langle 001\rangle$ direction. The trace of the magnetization in the conical phase is shown by the yellow line in Fig. 2. When applying a magnetic field along the [001] axis, the magnetization in the conical phase is 

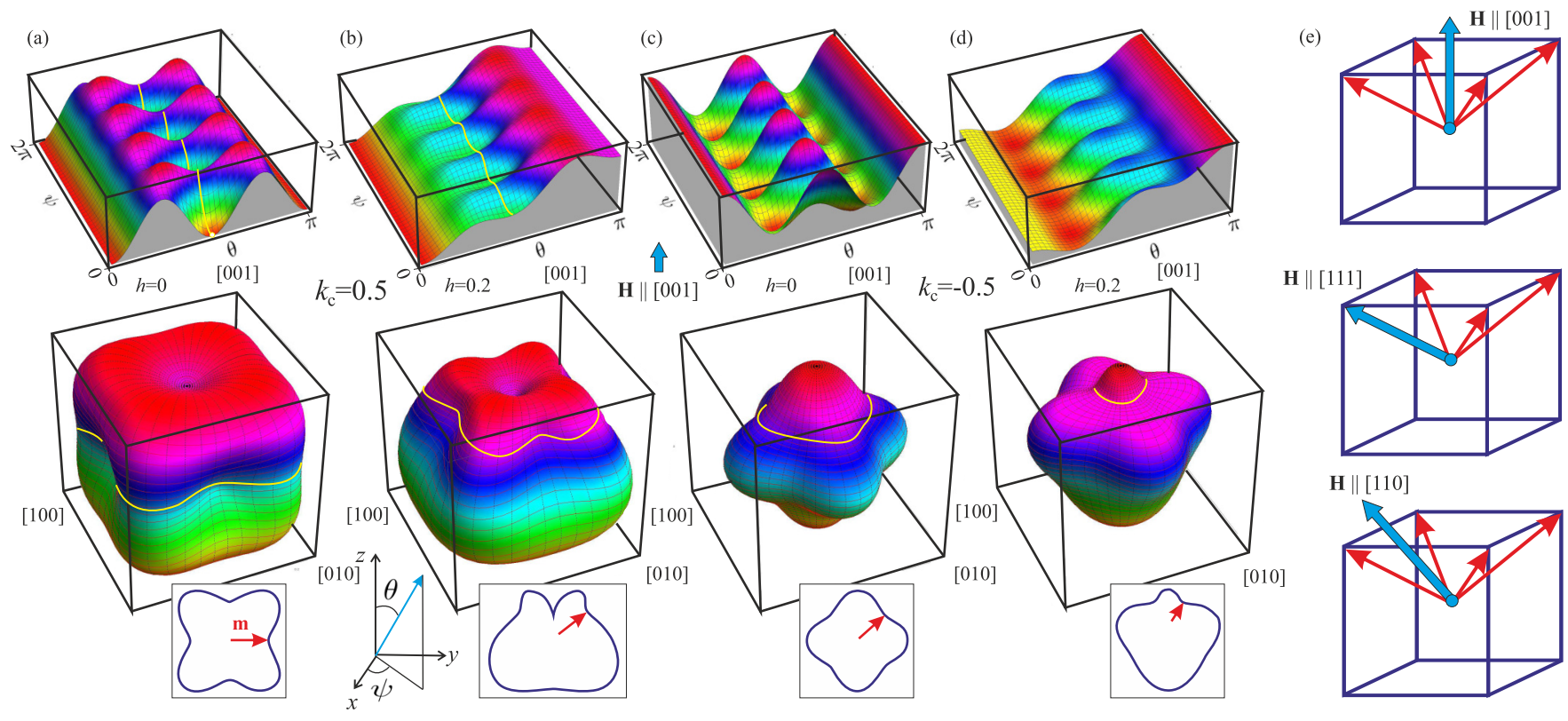

FIG. 2. Cubic anisotropy energy density (2) as a function of the polar and azimuthal angles $\theta$ and $\psi$ of the ferromagnetic state shown as twodimensional surfaces (first row) and three-dimensional polar plots (second row). The graphs in the third row depict cuts of the corresponding polar plots with $\psi=0$. The path of the rotating magnetization in the conical phase is shown by the yellow lines. The field is applied along the [001] direction. (a) and (b) correspond to $k_{c}=0.5$ and in this case, the states of the magnetization in the cone correspond only to the local minima. (c) and (d) correspond to $k_{c}=-0.5$ and the magnetization rotates to sweep the global minima of the cubic anisotropy. (e) Reorientation of the spirals into the conical state for $k_{c}<0$ and different directions of the field. q-vectors of spirals are shown by red arrows and point along $\langle 111\rangle$ crystallographic axes (see text for details).

forced to rotate in the vicinity of hard $\langle 111\rangle$ axes, as shown in Fig. 2(b) for $h=0.2$. This underlies the subsequent first-order phase into the ferromagnetic state, which becomes evident by, e.g., the magnetization jumps in the magnetization curves shown in Fig. 1(e). In Fig. 1(d) we plot the difference between the energy densities of the conical spiral and the ferromagnetic states. In this plot the point $B$ for $k_{c}=0.1$ stands for the first-order phase transition that takes place when the energy difference between the two phases vanishes. Logically the same situation should also occur for $h \|[111]$ and $k_{c}<0$, where three hard anisotropy axes $\langle 001\rangle$ impose distortions to the conical phase and underlie the magnetization jump into the ferromagnetic state.

For $k_{c}<0$ and $h=0$ [Figs. 2(c) and 2(d)], the equilibrium states of the cubic anisotropy energy correspond to the $\langle 111\rangle$-type crystallographic directions. Thus, the maxima of the cubic anisotropy are the hard $\langle 001\rangle$-type directions, and if a magnetic field is applied along a $\langle 001\rangle$ axis, the angle between the easy directions and the field is $70.6^{\circ}$. In this case, the magnetization in the conical phase rotates so that it sweeps the easy directions $\langle 111\rangle$ [Fig. 1(b)]. Even at zero field the magnetic structure is conical; thus, it has a nonzero uniform magnetization [see magnetization curves in Fig. 1(g)]. However, this low-field conical phase is in reality a metastable solution overshadowed by the oblique spiral states with $\mathbf{q}$-vectors along the easy $\langle 111\rangle$-type directions. In a finite magnetic field the global minima of the cubic anisotropy gradually approach the field direction and thus display a second-order phase transition to the ferromagnetic state [Fig. 2(d)]. The same principle is valid also for $\mathbf{h}||[111]$ and $k_{c}>0$, where the angle between the field direction and the easy $\langle 001\rangle$ axes is $54.7^{\circ}$, whereas for the hard $\langle 111\rangle$ axes the angle is $70.6^{\circ}$. In this case the topology of the corresponding polar plots would allow the gradual alignment of the magnetization along the hard $\langle 111\rangle$ direction with increasing magnetic field.

\section{Conical domains in the ferromagnetic state}

As a consequence of the first-order phase transition that occurs for either $k_{c}>0$ and $h \|[001]$ or $h \|[111]$ and $k_{c}<0$, domains of the conical phase may persist in the field polarized state adopting the configuration illustrated in Fig. 3. Such domains exhibit both types of the rotational sense at their boundaries. At the left side of the depicted conical droplet [Figs. 3(a)-3(c)] the magnetization in the conical phase adjusts to the homogeneous state with the "correct" twist supported by the DMI. This leads to energy gain as shown by the negative energy density difference in the $x y$ plane, i.e., for a cross section perpendicular to the magnetic field $\mathbf{h}$ and the conical $\mathbf{q}$-vector, shown in Fig. 3(d). At the right side of the conical droplet, the magnetization acquires the "wrong" in-plane twist, which results in an energetic "penalty," as shown in Fig. 3(d).

A similar behavior is also found along the $z$ direction, which is parallel to $\mathbf{h}$ and q. As illustrated in Figs. 3(e) and 3(f) the energy density of the conical droplet exhibits alternating negative and positive contributions corresponding to "correct" and "wrong" senses of the magnetization rotation at the boundary to the field polarized state. When the magnetic field is increased from the conical to the ferromagnetic phase, the conical droplet may either continuously expand for $h<h_{B}$, 

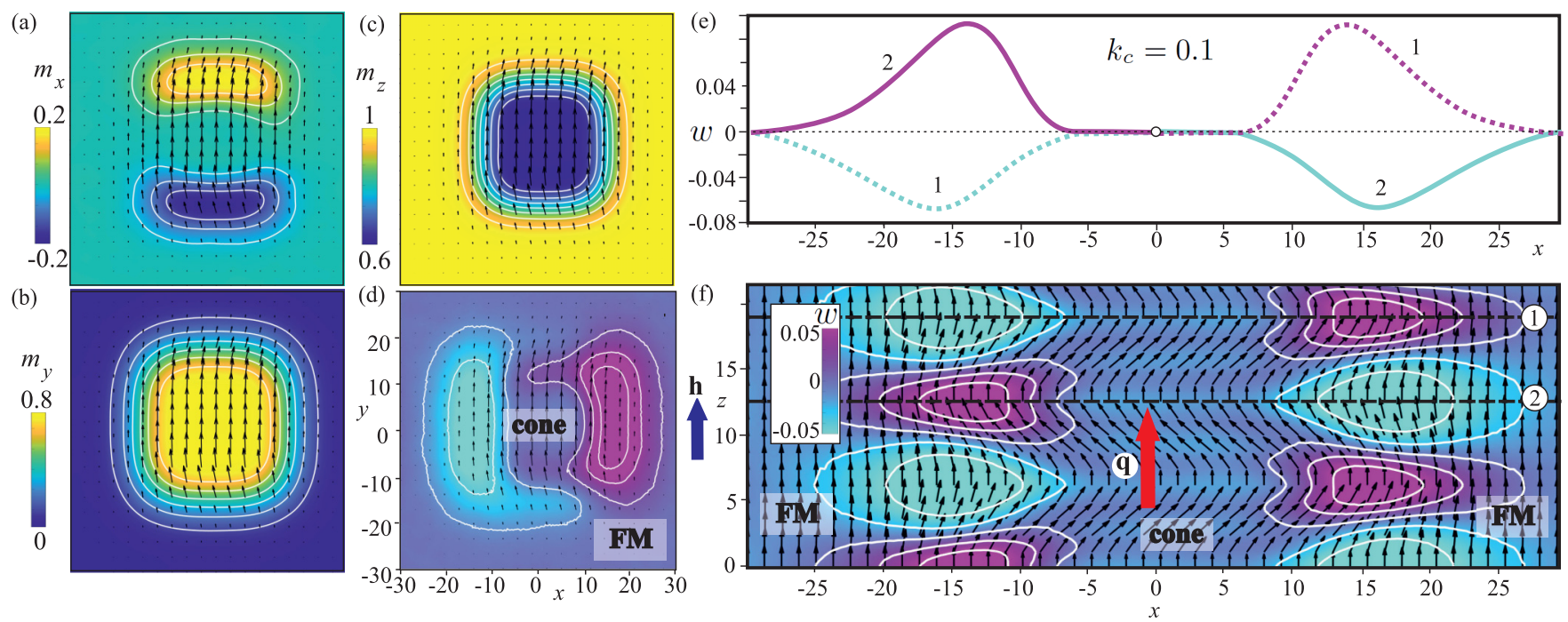

FIG. 3. Internal structure of conical droplets formed during the first-order phase transition from the conical to the ferromagnetic state shown as color plots of the components of the magnetization (a)-(c) and the energy density $w$ (d). The black arrows in (a)-(d) are projections of the magnetization onto the $x y$ plane, i.e., the same for all four panels. The q-vector of the conical phase as well as the applied magnetic field $\mathbf{h}$ are directed along $z$. The energy density in the $x z$ cross section of the conical droplet (f) exhibits alternating parts with the negative and positive contributions corresponding to "correct" and "wrong" senses of the magnetization rotation at the boundary to the ferromagnetic state. (e) shows the corresponding linear cross sections along $x$ marked by the dashed lines 1 and 2 in (f).

as seen in Fig. 1(d), or gradually shrink for $h>h_{B}$, a feature that underlies the kinetic character of such first-order phase transition. Remarkably, when the magnetic field is decreased from the field polarized to the conical state, the energy density shown in Fig. 3(f) leads to an "in-phase" merge of neighboring conical droplets that initially may be formed with some phase shift with respect to each other. Then, the region with the positive energy density at the boundary of one conical droplet is annihilated by the corresponding part with the negative energy of another droplet, a feature that underlies in-phase "zipping" and "unzipping" of conical droplets.

\section{B. The helical-to-conical phase transition at the critical field $\boldsymbol{H}_{c 1}$}

In this section we present qualitative results on the spiral reorientation processes that occur for $k_{c}<0$ and for different directions of the applied magnetic field [Fig. 2(e)]. Numerically exact solutions for the spiral states are obtained by direct minimization of the energy functional, given by Eq. (1), including $K_{c}$ in Eq. (2), and are discussed elsewhere [28]. Since $k_{c}>0$ favors easy axes of $\langle 111\rangle$ type, the ground state at zero field consists of domains of spiral states with the corresponding directions of the q-vectors. Figure 2(d) shows a schematic layout of the $\mathbf{q}$-vectors in stable and metastable spiral states with respect to the field and the crystallographic directions.

For $\mathbf{H} \|[001$ ] [first panel in Fig. 2(e)], all four equivalent spiral states with $\mathbf{q} \|\langle 111\rangle$ are at an angle of $35.2^{\circ}$ with respect to the field. As also discussed in Ref. [28], with increasing magnetic field, these wave vectors first rotate gradually and then jump by a first-order phase transition toward the field direction.

For $\mathbf{H} \|[111]$ [second panel in Fig. 2(e)], only the spiral that is co-aligned with the magnetic field is stable, whereas the spirals along the other equivalent wave vectors $\mathbf{q} \|\langle 111\rangle$ become metastable. With decreasing magnetic field only one spiral (or conical) domain with the q-vector along the field persists and at zero field the other spiral domains are not recovered.

For $\mathbf{H} \|[110]$ [third panel in Fig. 2(e)], there are two sets of $\langle 111\rangle$ directions composing smaller and larger angles with respect to the field. Thus the field-driven transition into the conical phase is a two-step process: the energetically unfavorable spiral states with the angle $90^{\circ}$ reorient first, whereas the stable spirals with the angle $27.35^{\circ}$ gradually rotate and eventually flip along the field. With decreasing magnetic field the transitions at both critical fields should occur in a reproducible manner, possibly with some hysteresis.

\section{Phase transition between two elliptically distorted conical states for $\mathbf{H}||[110]$}

For $\mathbf{H} \|[110]$, independently of the sign of the cubic anisotropy constant $k_{c}$, the $\mathbf{q}$-vector of the conical state is surrounded by two hard and two easy anisotropy axes $\langle 111\rangle$ and $\langle 100\rangle$. This rosette of anisotropy axes leads to the characteristic elliptical distortion of the conical state, which moreover changes when the magnetization rotation occurs in the exterior or interior of the rosette. In particular for $k_{c}<0$ and low field values, the trajectory of the rotating magnetization is elongated in-plane along the hard $\langle 100\rangle$ axes [Figs. 4(a) and 4(b)]. With increasing magnetic field the magnetization approaches the rosette, penetrates the rosette's interior, and changes its elliptical distortion being now elongated along the easy $\langle 111\rangle$ axes [Figs. 4(c) and 4(d)].

For $k_{c}>0$, the same process takes place, but with inverse switch of the elliptical distortion (Fig. 5). The ratios of the ellipse major and minor axes for both signs of the anisotropy constant are plotted as a function of the magnetic field for different values of $k_{c}$ in Figs. 4(e) and 5(e). From these 

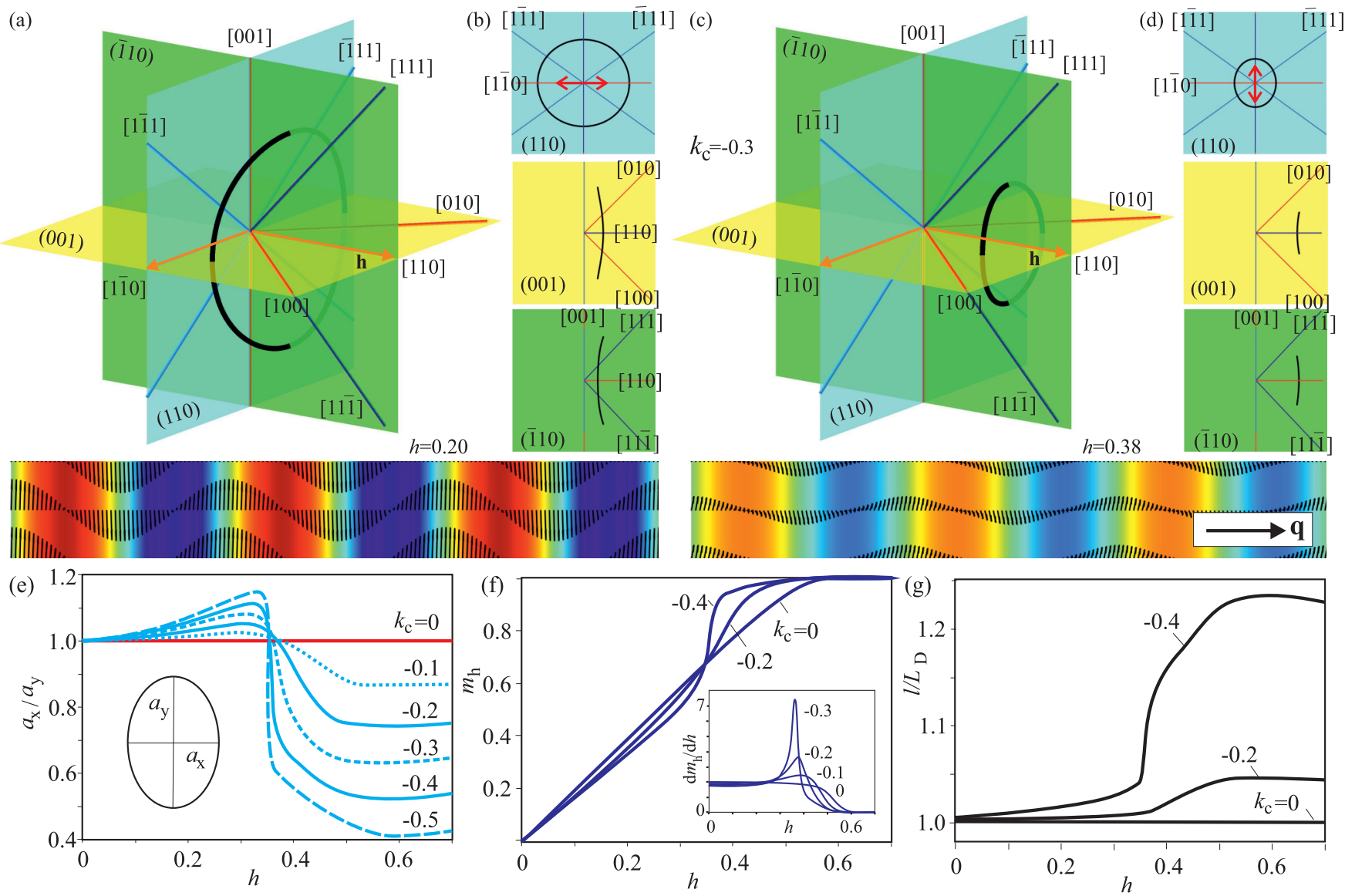

FIG. 4. $\mathbf{h} \|[110], k_{c}=-0.3$. Trajectories of the magnetization rotation in the conical phase (a), (c) in the presence of cubic anisotropy are depicted by the black curves for two values of the field, $h=0.2$ (a), $h=0.38$ (c). The anisotropy axes are represented by the red straight lines for the $\langle 100\rangle$-type axes, and the blue lines for the $\langle 111\rangle$-type axes. The corresponding projections of the magnetization traces on the planes (110), (001), (110) are shown in (b) and (d), correspondingly, and reveal the change of ellipticity, which is also characterized by the ratio of ellipse half-axes in (e). Such an ellipticity change is accompanied by magnetization jumps and peaks in the magnetic susceptibility, as shown in (f) and its inset. For small anisotropy constants the periodicity changes only by a few percent (g). The supplemental video is available at Ref. [29].

curves as well as from the magnetization curves [Fig. 4(f)], it becomes clear that the phase transition between these two conical phases is more abrupt for the negative values of $k_{c}$.
This might be explained by the different angles the easy and hard anisotropy axes compose with respect to the field- $35.3^{\circ}$ for $\langle 111\rangle$ and $45^{\circ}$ for $\langle 100\rangle$. Indeed, when $k_{c}<0$, the rotating

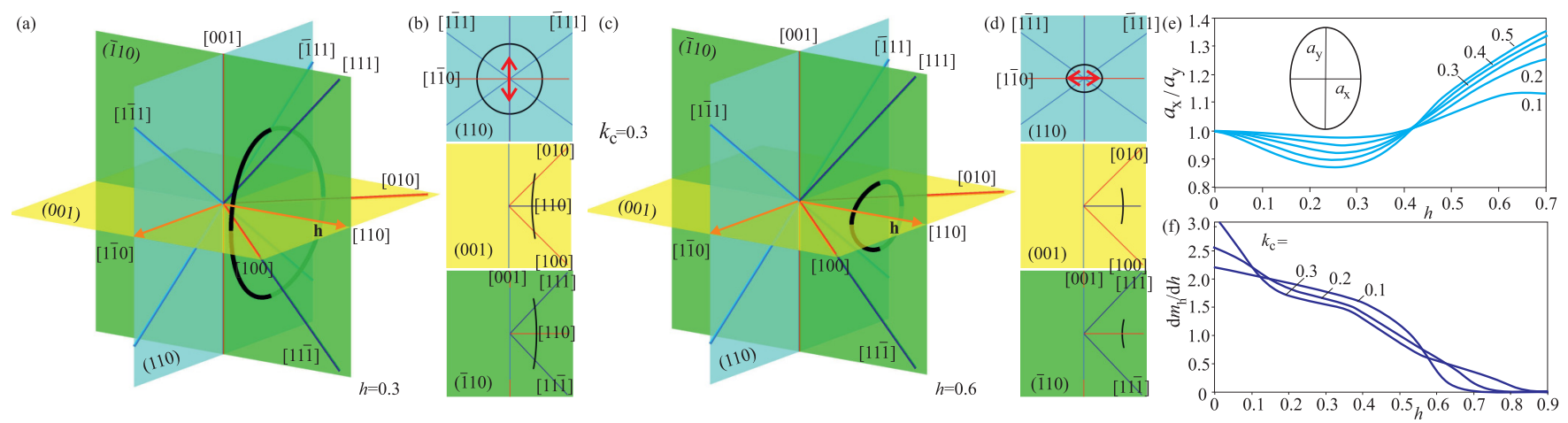

FIG. 5. $\mathbf{h} \|[110], k_{c}=0.3$. Trajectories of the magnetization rotation in the conical phase (a), (c) are shown for $h=0.3$ (a), $h=0.6$ (c). The corresponding projections of the magnetization traces in (b) and (d) demonstrate a reverse ellipticity change as compared with Fig. 4 . This is also indicated by the ratio of ellipse half-axes in (e) showing first decrease and then a subsequent increase with the field. Interestingly, the characteristic fingerprints of the ellipticity change are not detected at the magnetic susceptibility (f). The supplemental video is available at Ref. [29]. 

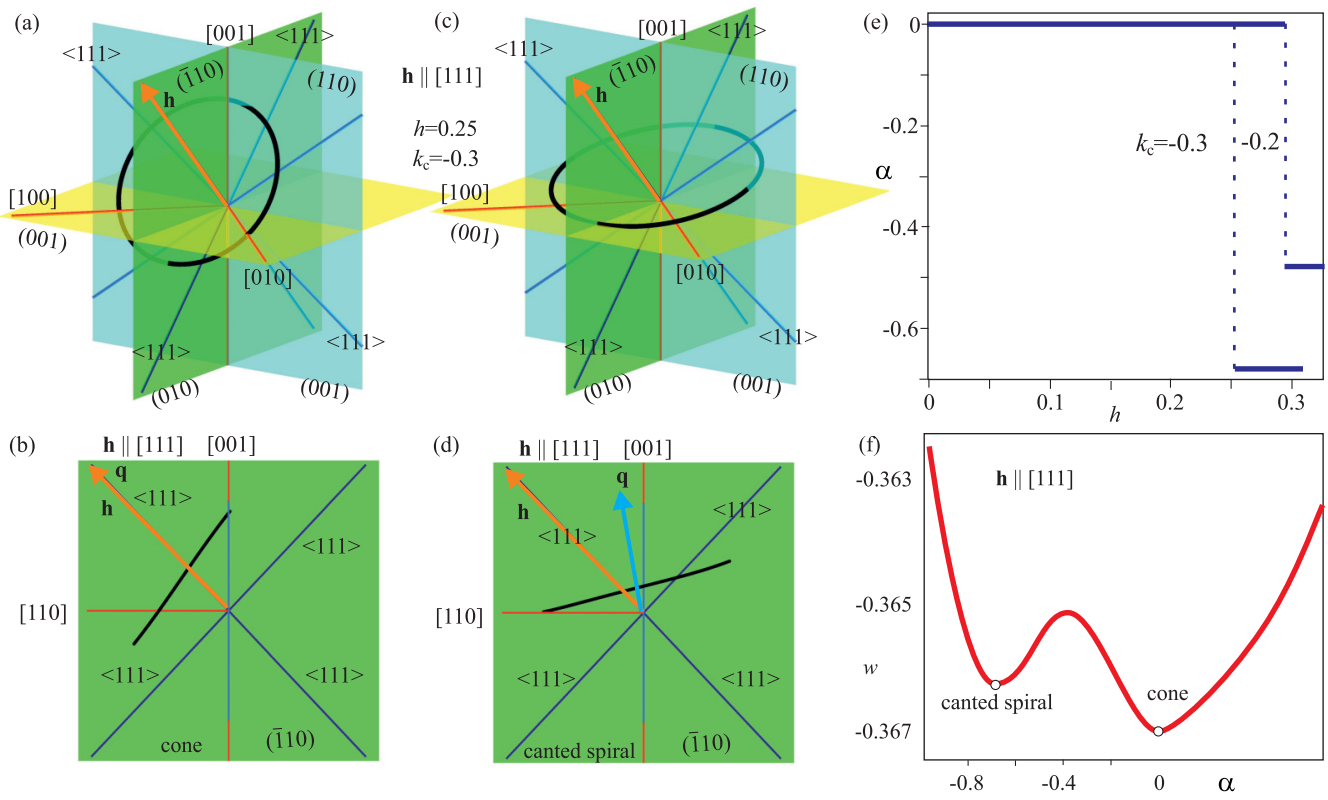

FIG. 6. Spiral reorientation near the saturation field $h_{c 2}$, for $\mathbf{h}||[111]$ and $k_{c}<0$. As seen from the sketches (a), (b), the magnetization in the conical state embraces two easy axes of the cubic anisotropy and is favored by the Zeeman energy. In the canted spiral state (c), (d), the magnetization attempts to embrace all four easy $\langle 111\rangle$ axes, at the expense of the Zeeman energy. This is a first-order phase transition as seen from the energy density (f), for $\mathbf{q}$ varied in the plane (010). The deflection angle $\alpha$ of the wave vector $\mathbf{q}$ with respect to the field, shown in (e), depends on the value of $k_{c}$ and tends to $70.6^{\circ}$, thus to $\mathbf{q} \|[001]$ in the present configuration.

magnetization tries to avoid two hard axes [100] and [010] and this results in the jump of the magnetization from the rosette's exterior to its interior. For $k_{c}>0$ [100] and [010] axes are easy anisotropy directions. Thus, although the sense of ellipticity changes, no jump of the magnetization is observed. An important point is that the phase transition between the two different conical states leaves the periodicity of the spiral state almost unaffected. In fact, the period changes just by a few percent for low values of the cubic anisotropy [less than $4 \%$ in Fig. 4(g) for $k_{c}=-0.2$ ], and this change is hardly detectable by, e.g., neutron scattering experiments. The field dependence of $d m_{h} / d h$ [Fig. 4(f), inset, and Fig. 5(f)], on the contrary, provides a clear hint for this transition as it shows a $\lambda$-shaped spike only for $k_{c}<0$.

\section{Spiral flip near the saturation field $\boldsymbol{H}_{c 2}$}

In the following we will show that spiral reorientation processes akin to those at $H_{c 1}$ may intervene also near the saturation field $H_{c 2}$. Jumps of the spiral q-vectors are less expected although they may be realized for relatively moderate values of the cubic anisotropy constant (Fig. 6). Indeed, the conical phase co-aligned with the field is considered to be the most favorable state that benefits from the Zeeman interaction. In the following, we show theoretically that a tilted spiral state may originate from the interplay between Zeeman and cubic anisotropy interactions, which is generic to chiral magnets, and thus may be important for explaining the experimental data of specific cubic helimagnets. The spiral jumps are also sensitive to the direction of the applied magnetic field and the value of $k_{c}$, since relatively large values of $k_{c}$ are needed to implement particular reorientations.
As an illustrative example, we consider the case with $\mathbf{H}||[111]$ and easy $\langle 111\rangle$ axes of the cubic anisotropy (Fig. 6). The q-vector undergoes a first-order transition from the conical state [Figs. 6(a) and 6(b)] into a tilted spiral state with almost constant tilt angle for a range of magnetic field values [Figs. 6(c) and 6(d)], with both the tilt angle and the field range dependent on $k_{c}$. The sketches in Figs. 6(c) and 6(d) are instructive to deduce the nature of this tilted spiral state. Indeed, in an increasing magnetic field, the magnetization in the conical state sweeps only two $\langle 111\rangle$ anisotropy axes [Figs. 6(a) and 6(b)]. In the tilted state, however, by sacrificing the Zeeman energy, the magnetization may try to embrace all four $\langle 111\rangle$ axes [Figs. 6(c) and 6(d)]. This leads to the maximum tilt angle $70.6^{\circ}$, reached for the higher $k_{c}$ values [Fig. 6(e)]. This phase transition is accompanied by characteristic jumps in the magnetization curve. An interesting point is that such a reorientation phase transition requires a threshold value of $k_{c}$. For $k_{c}=-0.1$ not even a hint of the metastable tilted spiral becomes apparent in the angle-dependent energy density [like that in Fig. 6(f)].

Conceptually the same reorientation process may occur also for $\mathbf{H} \|[110]$.

\section{OBLIQUE SPIRAL STATES DUE TO THE COMPETING ANISOTROPY INTERACTIONS}

Tilted spiral states also may originate from the interplay between competing anisotropic spin interactions, which is generic to chiral magnets. In fact, such an interplay gives a rather good control over oblique states with smooth variation of the tilt angle. As an instructive example, we consider spiral reorientation processes due to the competition between 

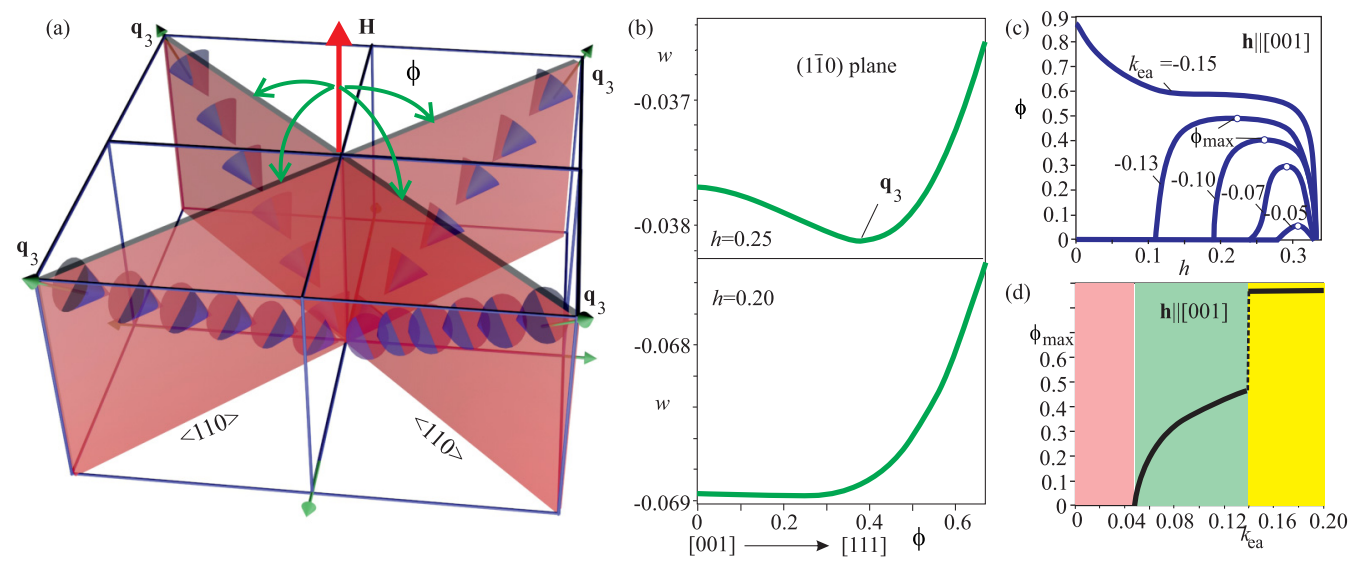

FIG. 7. Schematics of the tilted-spiral state that appears above a critical value of the magnetic field, in which the wave vector $\mathbf{q}$ rotates away from the magnetic field $\mathbf{h}||[001]$ toward the $\langle 111\rangle$ directions. (b) Energy density plotted as a function of the tilt angle $\phi$ in the (110) plane for $h=0.2$ (lower panel) and $h=0.25$ (upper panel) exhibits the minimum shift. (c) Magnetic field dependence of the angle $\phi$ for different values of $k_{e a}$. Above the critical value, $k_{e a} \approx 0.05, \phi$ increases from 0 , which corresponds to the conical spiral phase, to a maximal value, $\phi_{\max }$ (empty circle), and then decreases back to 0 as the magnetic field strength, $h$, increases. For $k_{e a}>0.14$, q $\|\langle 111\rangle$ even at zero field and smoothly rotates toward [001] with increasing field. (c) Dependence of the maximal tilt angle $\phi_{\max }$. For $k_{e a}<0.05$ (red area), $\phi_{\max }=0$, which implies that $\mathbf{q} \|[001]$. By increasing the strength of the anisotropic exchange, $\mathbf{q}$ smoothly rotates toward $\langle 111\rangle$ (green area), and for $k_{e a}>0.14$, $\mathbf{q}||\langle 111\rangle$ (yellow area).

cubic anisotropy $k_{c}$ with the easy axes $\langle 100\rangle$ and exchange anisotropy $k_{e a}<0$, i.e., with easy $\langle 111\rangle$ axes.

According to the symmetry analysis of the $P 2{ }_{1} 3$ structure of cubic helimagnets done by Bak and Jensen [30] and by Nakanishi [31], such an exchange anisotropy has the following form:

$$
w_{e a}=k_{e a}\left[\left(\frac{\partial m_{x}}{\partial x}\right)^{2}+\left(\frac{\partial m_{y}}{\partial y}\right)^{2}+\left(\frac{\partial m_{z}}{\partial z}\right)^{2}\right] .
$$

The constant $k_{\text {ea }}$ is typically one order of magnitude smaller than the exchange stiffness $A$. The role of the energy term (3) manifests itself in fixing the propagation directions of spiral states, e.g., as suggested by Bak and Jensen for MnSi [30], similarly to the cubic magnetocrystalline anisotropy. Therefore, the exchange and cubic anisotropy may be considered as a third level of hierarchy of energy scales following the exchange and DM interactions.

For the present setup of easy anisotropy axes, the tilted spiral state is found only for $\mathbf{h} \|[001]$. Above a critical field value, the conical spiral [Figs. 1(a) and 2(b)] begins to tilt toward one of the four body diagonals, the $\langle 111\rangle$ directions, as shown in Fig. 7(a). Such a tilted spiral state appears when $\left|k_{e a}\right|$ exceeds a critical value, which is slightly lower than 0.05 for $k_{c}=0.1$. Figure 7(b) shows the dependence of the spiral energy on the tilt angle $\phi$ with $\mathbf{q}$ varying in the (110) plane, for $h=0.2$ and $h=0.25$. As $h$ increases, $\phi$ grows, reaches its maximal value, and then decreases back to zero, corresponding to a return to the conical spiral state [Fig. 7(c)]. The maximal tilt angle, $\phi_{\max }$, which depends on the ratio of the competing fourth-order and exchange anisotropies, is plotted in Fig. 7(d). As the exchange anisotropy increases above the critical value, $\left|k_{e a}\right|>0.14$, the state with $\mathbf{q} \|\langle 111\rangle$ is stabilized even at zero magnetic field.

This effect can be understood as follows: the competing anisotropies give rise to new minima of the conical spiral energy in the q-space. If only the fourth-order anisotropy $k_{c}$ is taken into account, the global energy minimum for $\mathbf{H} \|\langle 001\rangle$ is obtained for $\mathbf{q} \| \mathbf{H}$. However, the additional anisotropy term in Eq. (2) with an appropriate sign of $k_{e a}$ shifts these minima away from [001] toward the four directions $\langle 111\rangle$ and thus underlies an instability of the conical state [Fig. 7(a)].

\section{OPEN QUESTIONS AND PERSPECTIVES}

In the following we briefly discuss the impact the spiral states may have on the properties of chiral skyrmions. On one hand, the behavior of spiral states can be considered from the point of view of skyrmion stability. In particular in bulk $\mathrm{Cu}_{2} \mathrm{OSeO}_{3}[12,13]$, cubic anisotropy was experimentally found to stabilize skyrmions in the form of a hexagonal skyrmion lattice (SkL) for low temperatures far from the region of the $A$ phase, but for specific directions of the applied magnetic field. In order to stabilize SkL, one should ensure that the cubic anisotropy deforms the ideal configuration of spiral states as considered in Figs. 2(a) and 2(b). Thus, the phase diagrams of states along these field directions exhibit vast areas with thermodynamically stable SkLs [13,20,28]. On the other hand, the internal properties of spiral states could be imprinted also on the functionalities of isolated chiral skyrmions.

\section{A. Spiral states and the internal structure of isolated skyrmions}

Indeed, isolated skyrmions acquire a magnetization distribution according to a surrounding "parental" or host state. In a state that is homogeneously magnetized along the field, axially symmetric skyrmion particles may form that underlie a repulsive interskyrmion potential [32]. On the other hand, when surrounded by a conical phase with the wave vector along the skyrmion axes, skyrmions develop a threedimensional structure: the central core region nearly preserves 
the axial symmetry, whereas the domain-wall region, which connects the core with the embedding conical state, acquires a crescent-like shape. This asymmetric profile of the cross section forms a screwlike modulation along the skyrmion axis, matching the rotating magnetization of the conical phase at all cross sections [33]. To reduce the energy excess necessarily related to such a rotation, isolated skyrmions tend to form compact clusters with the unimpeded magnetization rotation in their interior.

Experimentally, clusters of skyrmions have been observed in thin $(70 \mathrm{~nm})$ single-crystal samples of $\mathrm{Cu}_{2} \mathrm{OSeO}_{3}$ using transmission electron microscopy [34]. In a bulk $\mathrm{Cu}_{2} \mathrm{OSeO}_{3}$ for $\mathbf{H} \|[001]$, skyrmion clusters surrounded by the conical state exist up to the field $H_{c 2}$ of the first-order phase transition between the cone and the ferromagnetic state Figs. 2(a) and 2(b). With the onset of the homogeneous state, the interskyrmion attraction turns into repulsion and the constituent skyrmions disperse. If the number of isolated skyrmions is sufficiently high, they may form a SkL with an equilibrium period that exists above the $H_{c 2}$ line, as observed experimentally $[12,13]$. However, if the number of skyrmions is low, they keep moving away from each other and the skyrmion signal in the experiment should fade away with time. Thus, the following questions related to the skyrmion structure and skyrmion-skyrmion interaction arise: (i) What would be the impact of the elliptically distorted conical states for $\mathbf{H}||[110]$ (Figs. 4 and 5) or the oblique spiral states (Fig. 7)? Does the skyrmion attraction become anisotropic in these cases? (ii) How does the structure of skyrmion clusters distort during the spiral flips in Fig. 6? Do skyrmions avert this sort of spiral jump or are they instead annihilated via Bloch points?

\section{B. Spiral states as a background to accommodate differently oriented skyrmions}

Recently it was found that skyrmion tubes within the conical phase may orient either along or perpendicular to a wave vector $[35,36]$. The first type of skyrmions perfectly blends into the homogeneously saturated state after the cone closes in a strong magnetic field either by the first-order phase transition [Fig. 2(b)] or by the second-order one [Fig. 2(d)]. The second type of skyrmion perfectly blends into the spiral state and represents two merons with equally distributed topological charge $Q=1 / 2$. Then, a crossover between the two types of isolated skyrmions occurs for an intermediate value of an applied magnetic field. Thus, the found oblique spiral states (Figs. 6 and 7) may underlie new skyrmion states oblique with respect to the field and their controllable switch by the field.

\section{Spiral states and the nucleation of skyrmions}

Skyrmion nucleation may occur via different mechanisms specified by the stable and metastable spiral states $[13,14]$.
In particular, the low-temperature stable $\mathrm{SkL}$ in the bulkinsulating cubic helimagnet $\mathrm{Cu}_{2} \mathrm{OSeO}_{3}$ for $\mathbf{H}||[001]$ may appear via rupture formation of spiral states with wave vectors perpendicular to the field [13], along [100] and [010]. In this rather conventional mechanism also observed in the first papers on skyrmion visualization [6,7], the pairs of merons behave as if they were free particles. The properties of such skyrmions and merons within the spiral background were recently investigated in Refs. [37,38]. The essential difference in this case is that the spiral states are metastable solutions, but give rise to a stable SkL.

The experimental findings in $\mathrm{Cu}_{2} \mathrm{OSeO}_{3}$ [13] also hint that skyrmions may appear from the conical and the homogeneous states near the critical field $H_{c 2}$. This may occur via formation of magnetic torons of finite length accompanied by their elongation due to the cubic or exchange anisotropy, which leads to the negative energy density of skyrmion cores and their mutual attraction. Such a homogeneous nucleation mechanism via torons-spatially localized three-dimensional particles composed of a skyrmion filament of finite length cupped by two Bloch points terminating its prolongationwas recently discussed in Refs. [15,39]. In this sense, all sorts of the magnetization inhomogeneities provided, e.g., by the domain walls between spiral domains near the flip transition (Fig. 6) or by the superstructure formed from the rotating oblique spiral (Fig. 7), represent a favorable medium for the nucleation and stabilization of skyrmions.

\section{CONCLUSIONS}

To conclude, we have considered the magnetic-field-driven reorientation transitions of spiral states that occur at the critical fields $H_{c 1}$ and $H_{c 2}$. We have shown that the theoretical model (1) predicts richer phase diagrams that are much more complex than assumed so far. Novel phases are stabilized, such as an oblique spiral state that has recently been reported for the bulk helimagnet $\mathrm{Cu}_{2} \mathrm{OSeO}_{3}[11,12]$. Moreover, phase transitions related to the ellipticity switch of spiral states may occur like the one that has been theoretically predicted for $H||[110]$ but has not been yet observed experimentally. Our theoretical findings can thus serve as a guideline for planning future experiments that would systematically explore the role of impact of specific geometries of easy and hard anisotropy axes on the ground states of chiral helimagnets.

\section{ACKNOWLEDGMENTS}

The authors are grateful to Christos Panagopoulos, Sandor Bordacs, Ulrich Rößler, and Maxim Mostovoy for useful discussions. C.P. acknowledges financial support from the Vrije FOM program "Skyrmionics." This work was funded by DFG Priority Program SPP2137, Skyrmionics, under Grant No. KE 2370/1-1. A.O.L. thanks Ulrike Nitzsche for technical assistance.
[1] I. E. Dzyaloshinskii, Theory of helicoidal structures in antiferromagnets. I. Nonmetals, Zh. Eksp. Teor. Fiz. 46, 1420 (1964) [Sov. Phys. JETP 19, 960 (1964)].
[2] Y. Ishikawa and M. Arai, Magnetic phase diagram of MnSi near critical temperature studied by neutron small angle scattering, J. Phys. Soc. Jpn. 53, 2726 (1984). 
[3] K. Kadowaki, K. Okuda, and M. Date, Magnetization and magnetoresistance of MnSi, J. Phys. Soc. Jpn. 51, 2433 (1982).

[4] Yu. A. Izyumov, Modulated, or long-periodic, magnetic structures of crystals, Sov. Phys. Usp. 27, 845 (1984).

[5] S. Mühlbauer, B. Binz, F. Jonietz, C. Pfleiderer, A. Rosch, A. Neubauer, R. Georgii, and P. Böni, Skyrmion lattice in a chiral magnet, Science 323, 915 (2009).

[6] X. Z. Yu, Y. Onose, N. Kanazawa, J. H. Park, J. H. Han, Y. Matsui, N. Nagaosa, and Y. Tokura, Real-space observation of a two-dimensional skyrmion crystal, Nature (London) 465, 901 (2010).

[7] X. Z. Yu, N. Kanazawa, Y. Onose, K. Kimoto, W. Z. Zhang, S. Ishiwata, Y. Matsui, and Y. Tokura, Near room-temperature formation of a skyrmion crystal in thin-films of the helimagnet FeGe, Nat. Mater. 10, 106 (2011).

[8] D. Liang, J. P. DeGrave, M. J. Stolt, Y. Tokura, and S. Jin, Current-driven dynamics of skyrmions stabilized in $\mathrm{MnSi}$ nanowires revealed by topological Hall effect, Nat. Commun. 6, 8217 (2015).

[9] A. B. Butenko, A. A. Leonov, A. N. Bogdanov, and U. K. Rößler, Theory of vortex states in magnetic nanodisks with induced Dzyaloshinskii-Moriya interactions, Phys. Rev. B 80, 134410 (2009).

[10] H. Du, D. Liang, C. Jin, L. Kong, M. J. Stolt, W. Ning, J. Yang, Y. Xing, J. Wang, R. Che, J. Zang, S. Jin, Y. Zhang, and M. Tian, Electrical probing of field-driven cascading quantized transitions of skyrmion cluster states in $\mathrm{MnSi}$ nanowires, Nat. Commun. 6, 7637 (2015).

[11] F. Qian, L. J. Bannenberg, H. Wilhelm, G. Chaboussant, L. M. DeBeer-Schmitt, M. P. Schmidt, A. Aqeel, T. T. M. Palstra, E. H. Bruck, A. J. E. Lefering, C. Pappas, M. Mostovoy, and A. O. Leonov, New magnetic phase of the chiral skyrmion material $\mathrm{Cu}_{2} \mathrm{OSeO}_{3}$, Sci. Adv. 4, eaat7323 (2018).

[12] A. Chacon, L. Heinen, M. Halder, A. Bauer, W. Simeth, S. Muehlbauer, H. Berger, M. Garst, A. Rosch, and C. Peiderer, Observation of two independent skyrmion phases in a chiral magnetic material, Nat. Phys. 14, 936 (2018).

[13] L. J. Bannenberg, H. Wilhelm, R. Cubitt, A. Labh, M. Schmidt, E. Lelievre-Berna, C. Pappas, M. Mostovoy, and A. O. Leonov, Multiple low-temperature skyrmionic states in a bulk chiral magnet, npj Quantum Mater. 4, 11 (2019).

[14] A. O. Leonov and C. Pappas, Skyrmion clusters and conical droplets in bulk helimagnets with cubic anisotropy, Phys. Rev. B 99, 144410 (2019).

[15] A. O. Leonov and K. Inoue, Homogeneous and heterogeneous nucleation of skyrmions in thin layers of cubic helimagnets, Phys. Rev. B 98, 054404 (2018).

[16] A. Bauer and C. Pfleiderer, Generic aspects of skyrmion lattices in chiral magnets, in Topological Structures in Ferroic Materials, edited by J. Seidel (Springer International Publishing, Switzerland, 2016).

[17] A. Bauer, A. Chacon, M. Wagner, M. Halder, R. Georgii, A. Rosch, C. Pfleiderer, and M. Garst, Symmetry breaking, slow relaxation dynamics, and topological defects at the fieldinduced helix reorientation in MnSi, Phys Rev B 95, 024429 (2017).

[18] Y. Tokunaga, X. Z. Yu, J. S. White, H. M. Rønnow, D. Morikawa, Y. Taguchi, and Y. Tokura, A new class of chiral materials hosting magnetic skyrmions beyond room temperature, Nat. Commun. 6, 7638 (2015)
[19] A. N. Bogdanov and D. A. Yablonskii, Thermodynamically stable vortices in magnetically ordered crystals: Mixed state of magnetics, Zh. Eksp. Teor. Fiz. 95, 178 (1989) [Sov. Phys. JETP 68, 101 (1989)].

[20] A. O. Leonov, Twisted, localized, and modulated states described in the phenomenological theory of chiral and nanoscale ferromagnets, Ph.D. thesis, Technical University of Dresden, 2012.

[21] A. Bogdanov and A. Hubert, Thermodynamically stable magnetic vortex states in magnetic crystals, J. Magn. Magn. Mater. 138, 255 (1994).

[22] I. Kézsmárki, S. Bordacs, P. Milde, E. Neuber, L. M. Eng, J. S. White, H. M. Rønnow, C. D. Dewhurst, M. Mochizuki, K. Yanai, H. Nakamura, D. Ehlers, V. Tsurkan, and A. Loidl, Néeltype skyrmion lattice with confined orientation in the polar magnetic semiconductor $\mathrm{GaV}_{4} \mathrm{~S}_{8}$, Nat. Mater. 14, 1116 (2015).

[23] E. Ruff, S. Widmann, P. Lunkenheimer, V. Tsurkan, S. Bordacs, Istvan Kézsmárki, and A. Loidl, Multiferroicity and skyrmions carrying electric polarization in $\mathrm{GaV}_{4} \mathrm{~S}_{8}$, Sci. Adv. 1, e1500916 (2015).

[24] A. Butykai, S. Bordacs, I. Kézsmárki, V. Tsurkan, A. Loidl, J. Döring, E. Neuber, P. Milde, S. C. Kehr, and L. M. Eng, Characteristics of ferroelectric-ferroelastic domains in Néeltype skyrmion host $\mathrm{GaV}_{4} \mathrm{~S}_{8}$, Sci. Rep. 7, 44663 (2017).

[25] A. Butykai, D. Szaller, L. F. Kiss, L. Balogh, M. Garst, L. DeBeer-Schmitt, T. Waki, Y. Tabata, H. Nakamura, I. Kézsmárki, and S. Bordacs, Squeezing magnetic modulations by enhanced spin-orbit coupling of $4 d$ electrons in the polar semiconductor $\mathrm{GaMo}_{4} \mathrm{~S}_{8}$, arXiv:1910.11523.

[26] T. Kurumaji, T. Nakajima, V. Ukleev, A. Feoktystov, T.-h. Arima, K. Kakurai, and Y. Tokura, Néel-Type Skyrmion Lattice in the Tetragonal Polar Magnet $\mathrm{VOSe}_{2} \mathrm{O}_{5}$, Phys. Rev. Lett. 119, 237201 (2017).

[27] A. Leonov, U. K. Roessler, and A. N. Bogdanov, Phenomenological theory of magnetization reversal in nanosystems with competing anisotropies, J. Appl. Phys. 104, 084304 (2008).

[28] A. O. Leonov and C. Pappas, Multiple skyrmionic states and oblique spirals in bulk cubic helimagnets, in Magnetic Skyrmions and Their Applications, edited by Giovanni Finocchio and Christos Panagopoulos (Elsevier, to be published).

[29] See Supplemental Material at http://link.aps.org/supplemental/ 10.1103/PhysRevResearch.2.043386 for details on the elliptical distortions of conical states in video.

[30] P. Bak and M. H. Jensen, Theory of helical magnetic structures and phase transitions in MnSi and FeGe, J. Phys. C: Solid State Phys. 13, L881 (1980).

[31] O. Nakanishi, A. Yanase, A. Hasegawa, and M. Kataoka, The origin of the helical spin density wave in MnSi, Solid State Commun. 35, 995 (1980).

[32] A. O. Leonov, T. L. Monchesky, N. Romming, A. Kubetzka, A. N. Bogdanov, and R. Wiesendanger, The properties of isolated chiral skyrmions in thin magnetic films, New J. Phys. 18, 065003 (2016).

[33] A. O. Leonov, T. L. Monchesky, J. C. Loudon, and A. N. Bogdanov, Three-dimensional chiral skyrmions with attractive interparticle interactions, J. Phys.: Condens. Matter. 28, 35LT01 (2016).

[34] J. C. Loudon, A. O. Leonov, A. N. Bogdanov, M. C. Hatnean, and G. Balakrishnan, Direct observation of attractive skyrmions 
and skyrmion clusters in the cubic helimagnet $\mathrm{Cu}_{2} \mathrm{OSeO}_{3}$, Phys. Rev. B 97, 134403 (2018).

[35] A. O. Leonov, A. N. Bogdanov, and K. Inoue, Toggle-switchlike crossover between two types of isolated skyrmions within the conical phase of cubic helimagnets, Phys. Rev. B 98, 060411(R) (2018).

[36] H. R. O. Sohn, S. M. Vlasov, V. M. Uzdin, A. O. Leonov, and I. I. Smalyukh, Real-space observation of skyrmion clusters with mutually orthogonal skyrmion tubes, Phys. Rev. B 100, 104401 (2019).
[37] J. Müller, J. Rajeswari, P. Huang, Y. Murooka, H. M. Rønnow, F. Carbone, and A. Rosch, Magnetic Skyrmions and Skyrmion Clusters in the Helical Phase of $\mathrm{Cu}_{2} \mathrm{OSeO}_{3}$, Phys. Rev. Lett. 119, 137201 (2017).

[38] M. Ezawa, Compact merons and skyrmions in thin chiral magnetic films, Phys. Rev. B 83, 100408(R) (2011).

[39] G. P. Müller, F. N. Rybakov, H. Jónsson, S. Blügel, and N. S. Kiselev, Coupled quasimonopoles in chiral magnets, Phys. Rev. B 101, 184405 (2020). 